\title{
Application of GPR in extra-long tunnel engineering with a variety of adverse geological conditions
}

\author{
${ }^{a}$ M. GUO \& G. LIU, ${ }^{b}$ T.Y. BAI \& Z.P. LI \\ aSinohydro Bureau 14 CO., LTD. Chongqing, China \\ ${ }^{b}$ Key Laboratory of Ministry of Education for Efficient Mining and Safety of Metal Mine, University of \\ Science and Technology Beijing, Beijing, China
}

\begin{abstract}
KEYWORD: Ground penetrating radar; Extra-long tunnel; Geologic forecast
ABSTRACT: Geological disasters are always induced by excavating in extra-long tunnel engineering. In order to ensure safety in construction process, the ground penetrating radar (GPR) has been used to measure adverse geological conditions in front of the tunnel excavating face. Application experience of GPR in extra-long tunnel engineering with a variety of adverse geological conditions in southwest China was analyzed and shared, and GPR images of typical geological conditions were revealed to provide references for similar tunnel projects.
\end{abstract}

\section{INTRODUCTION}

In highway or high-speed railway engineering, the extra-long tunnel plays an irreplaceable role in crossing through complex geological and topographical, shortening the travelling distance and reducing environmental impact. Li Keqiang, premier of China, made it clear in report on the work of the government that 1.65 trillion Yuan would be invested into highway construction and about $30,000 \mathrm{~km}$ would be newly-built and rebuilt during the 13th national five-year plan. In southwest China, tunnel engineering is the key part in highway construction, and geologic forecast should be timely and accurate because of the long mileage and complicated geological conditions, thereby information of geological changes in front of the tunnel excavating face can be revealed. Furthermore, geologic forecast serves for tunnel construction and plays a key role in reducing and preventing the geological disasters.

In tunnel engineering, geological forecast process is taken by physical detection methods, and it is the forecast of various adverse geological conditions and geological disasters that may occur in front of the tunnel excavating face. The work for advance geological prediction is crucial to the tunnel information construction, reducing of blind construction and ensuring construction safety.The ground penetrating radar(GPR) method is a geophysical method which can determine the internal material distribution by use of high frequency radio waves, broadband electromagnetic wave propagates in the form of a pulse in the medium of rock and earth mass, and invisible objects or structure beneath the surface of soil can be detected. GPR has many advantages such as simple in operation, quick in measurement, uninterrupted in scanning and directly in results observing. In this paper, the application characteristics of GPR method in geological forecast in extra-long tunnel engineering of a variety of adverse geological conditions were in-depth studied, and the engineering practice of Lirang tunnel in Chongqing, China is combined to offer reference for similar tunnel construction projects.

\section{LIRANG TUNNEL PROJECT PROFILE}

Lirang tunnel is the key part of highway construction between two counties, Liangping and Zhong, in Chongqing, China. Lirang tunnel is an extra-long tunnel of 5517 meters in left and 5520 meters in right line. According to the information of preliminary geophysical prospecting, detailed geological survey and boreholes, adverse geological conditions in Lirang tunnel include karst, coal seam, gas, goaf and gyprock, and

(1) The tunnel passes through the karst valley, and was deeply affected by karst breccia and core of anticlines, as a result, the development of karst makes it possible that disasters such as roof failure, water and mud bursting occur; 
(2) The tunnel passes through the coal seam which is uneven in different areas and complex in thickness. Affected by geologic structure, the coal seam is extremely unstable, what's more, there are several coal mines and pit around the tunnel, during the construction process, it should be highly valued that the working out area and catchment and hazardous gas are dangerous;

(3) The tunnel passes through the stratum of gyprock, and the gyprock is highly corrosive to concrete and micro corrosive to steel bars, besides, the volume of plaster of paris will increase in the process of hydration, the high expansibility does harm to tunnel lining.

In the process of tunnel construction, especially the adverse geological sections, geological forecast is badly needed by use of GPR method.

\section{FUNDAMENTAL OF GPR}

In tunnel construction, the GPR is widely used in qualitative testing, geological hazard diagnosing and geological forecasting. Based on the propagation fundamental of pulsed electromagnetic waves underground, the GPR emits electromagnetic pulse by the transmitting antenna $\mathrm{T}$ and receives by the receiving antenna $R$ because that the pulsed waves are reflected by rock mass interface or geological abnormity body, then the recorded signals are transformed into images as shown in Figure 1. During the propagating through geological medium, the propagation path, electromagnetic field intensity and waveforms of electromagnetic waves change along with the electrical property and geometrical morphology of mediums, therefore based on the two-way travel time, amplitude and waveform characteristics, geological conditions of rock mass in front of the tunnel face can be forecasted, the formation interface and spatial structure of geological abnormity body can be figured out.

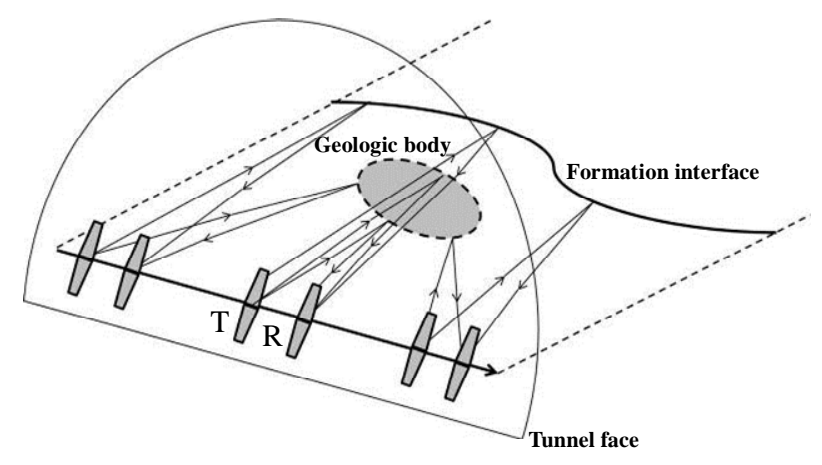

(a) Detecting diagrammatic sketch

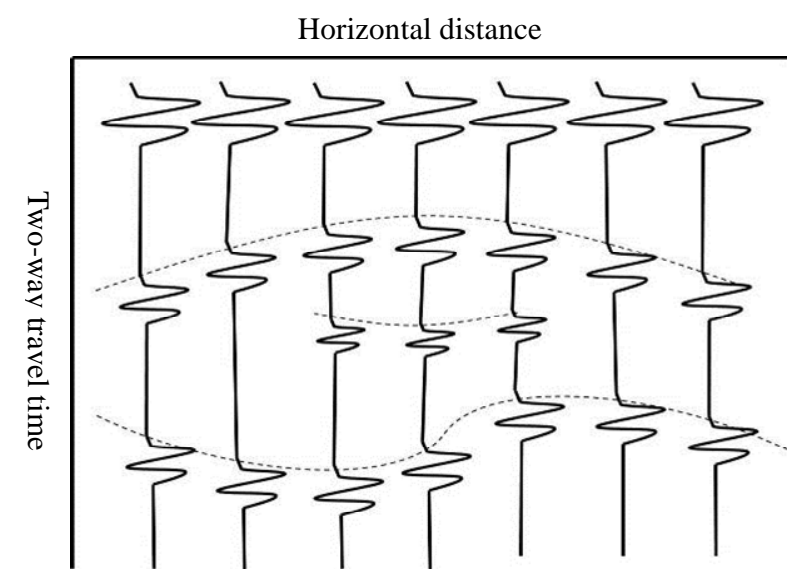

(b) GPR data image

Fig.1 Operating fundamental principle of GPR

\section{Propagation velocity of electromagnetic waves}

In the geological forecasting process, the GPR detects travel time of electromagnetic wave reflected by mediums or interfaces, in order to confirm location, the propagation velocity of electromagnetic wave should be calculated as:

$v=\frac{\omega}{\alpha}$ 
$\alpha=\omega \sqrt{\mu \varepsilon} \sqrt{\frac{1}{2}\left(\sqrt{1+(\sigma / \omega \varepsilon)^{2}}+1\right)}$

Where, $\omega$ is the angular frequency of electromagnetic wave, $\alpha$ is the phase coefficient, $\mu$ is the permeability, $\varepsilon$ is the dielectric constant and $\sigma$ is the specific conductance. Most of the rock medium are non-magnetic and non-conductive, then

$\frac{\sigma}{\omega \varepsilon} \quad 1$

And

$v=\frac{c}{\varepsilon_{r}}$

Where, $c$ is the propagation velocity of electromagnetic wave in vacuum, and $c=0.3 \mathrm{~m} / \mathrm{ns}, \varepsilon_{r}$ is the relative dielectric constant. It follows that velocity of electromagnetic wave $v$ depends on the relative dielectric constant of mediums.

Absorptive properties of electromagnetic waves

$\beta$ is the absorptive property which determines the decay rate of electromagnetic wave, the GPR works with high frequency and the displacement current counts much, therefore

$\beta=\frac{\sigma}{2} \sqrt{\frac{\mu}{\varepsilon}}$

$\beta$ is proportional to the specific conductance and inversely proportional to the square roof of the dielectric constant.

\section{Reflection and refraction of electromagnetic waves}

Electromagnetic waves propagates in various of geological conditions and will be reflected and refracted by interfaces with different wave impedance, and the energy of reflection depends on the reflectance, considering the detecting project to adverse geological body, the reflectance of $\mathrm{R}$ and $\mathrm{T}$ are

$$
\begin{gathered}
R=\frac{\sqrt{\varepsilon_{1}}-\sqrt{\varepsilon_{2}}}{\sqrt{\varepsilon_{1}}+\sqrt{\varepsilon_{2}}} \\
T=\frac{2 \sqrt{\varepsilon_{1}}}{\sqrt{\varepsilon_{1}}+\sqrt{\varepsilon_{2}}}
\end{gathered}
$$

Where, $\varepsilon_{1}$ and $\varepsilon_{2}$ are relative dielectric constant of mediums on and below the interface. The reflectance depends on the level of distinctness of relative dielectric constant in both sides of the medium interface, the higher the distinctness, the larger the reflectance, and more beneficial for detecting.

\section{Common geotechnical medium parameters in tunnel engineering}

In Lirang tunnel construction, based on the past project experience and field observation data ${ }^{[5]}$, several common geotechnical medium parameters are as follows in Table 1. 
Table 1 Common geotechnical medium parameters in tunnel

\begin{tabular}{ccccc}
\hline Medium & $\begin{array}{c}\text { Relative dielectric con- } \\
\text { stant }\end{array}$ & $\begin{array}{c}\text { Specific conduct- } \\
\text { ance } \\
\left(S \mathrm{~km}^{-1}\right)\end{array}$ & $\begin{array}{c}\text { Wave veloci- } \\
\text { ty } \\
\left(\mathrm{m} \mathrm{ns}^{-1}\right)\end{array}$ & $\begin{array}{c}\text { Attenuation coefficient } \\
\left(d B \mathrm{~m}^{-1}\right)\end{array}$ \\
\hline Air & 1.0 & 0.00 & 0.300 & 0.00 \\
Fresh water & 80.0 & 0.50 & 0.033 & 0.10 \\
Dry sand & $3.0-5.0$ & 0.01 & 0.150 & 0.01 \\
Saturated & $23.0-30.0$ & $0.10-1.00$ & 0.060 & $0.03-0.30$ \\
sand & $4.0-8.0$ & $0.50-2.00$ & 0.120 & $0.40-1.00$ \\
Limestone & $5.0-15.0$ & $1.00-100.00$ & 0.090 & $1.00-100.00$ \\
Mudstone & $5.0-15.0$ & $1.00-100.00$ & 0.090 & $1.00-100.00$ \\
Shale & $5.0-30.0$ & $1.00-100.00$ & 0.070 & $1.00-100.00$ \\
Quartz & $5.0-40.0$ & $2.00-100.00$ & 0.060 & $1.00-300.00$ \\
Clay & 6.4 & $1.00-100.00$ & 0.120 & $0.01-1.00$ \\
Concrete & & & & \\
\hline
\end{tabular}

\section{THE GPR METHOD USED IN LIRANG TUNNEL ENGINEERING}

\section{Field-data processing}

Based on the fundamental of GPR and field detecting data, after six process procedures of Static correction, Subtract-DC-shift, Energy decay gain, Subtracting average, Bandpass Butterworth filter and Running average, the typical GPR waveform characteristics can be obtained. In Figure 2, one trace was picked out to show the processing results of every single step.

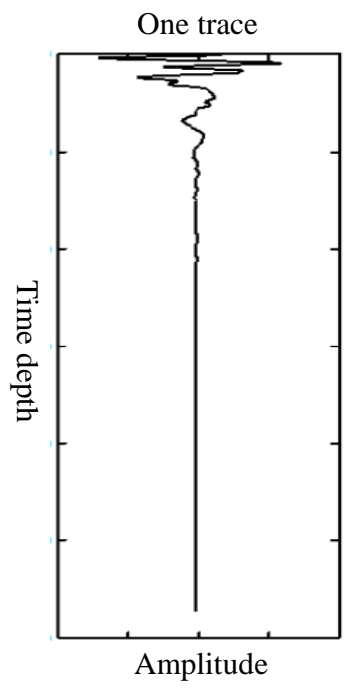

(a) Static correction

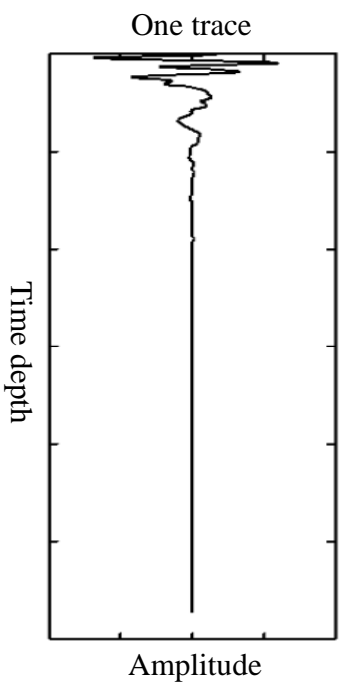

(b) Subtract-DC-shift

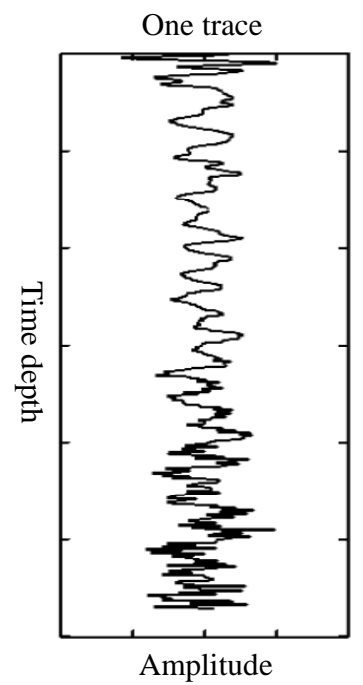

(c) Energy decay gain 


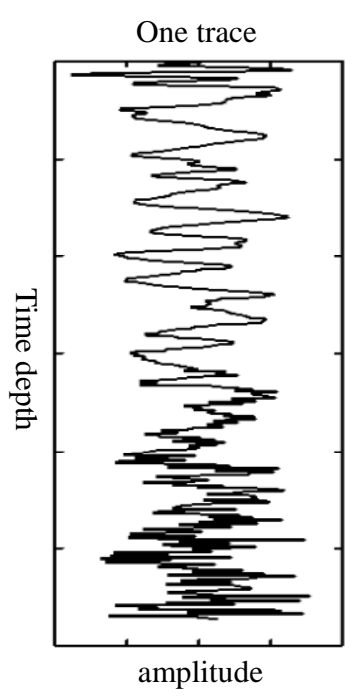

(d) Subtracting average

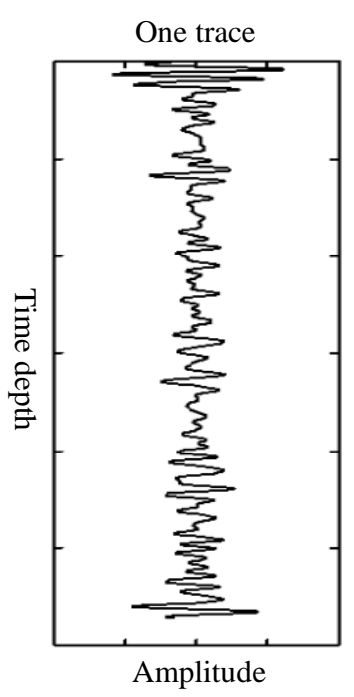

(e) Bandpass Butterworth filter

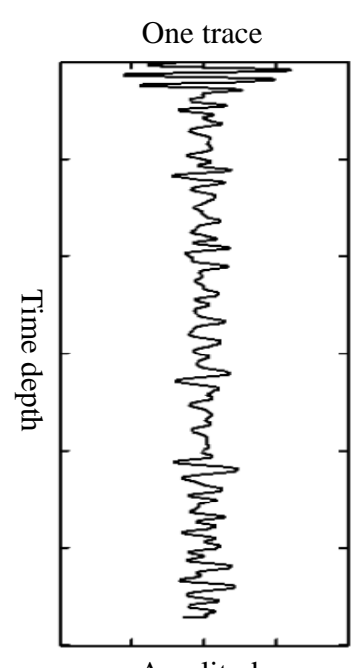

Amplitude

(f) Running average

\section{Detecting result of joint fissure rock mass}

Fig. 2 The GPR data processing procedures

As rock mass is cut by joint fissure, reflecting interfaces increase. If transverse joints develop clear, the reflected waves continue in same phase, and differ in amplitude and wave length with intact rock. While longitudinal joints develop clear, the energy of reflected waves change a lot and the frequency decrease, the reflected waves continue poor in same phase. With the increasing of the development of joint fissure, the GPR waves get more disordered. When the joint fissure develop with water and mud, the amplitude and wavelength of reflected waves change with the content of water or mud, and amplitude attenuates quickly, wavelength gets longer and notable in reflected waves difference on both sides of the interface.

In Lirang tunnel construction, the probing line of GPR is shown in Figure 3, adverse geology, hydrogeology, fault and its crushed zone, rock mass classification and stability can be predicted 0-20 meters long from the tunnel face. In Figure 4, typical joint fissure rock mass with water and mud was detected and transformed into GPR image, the amplitude and wavelength of reflected waves differ because of water content, and the amplitude attenuates quickly and wavelength gets longer, and the reflected waves discontinue in same phase.

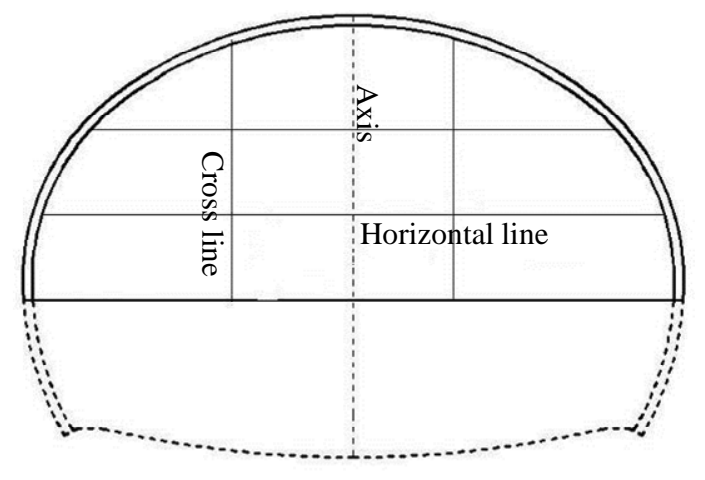

Fig.3 The probing line of GPR 


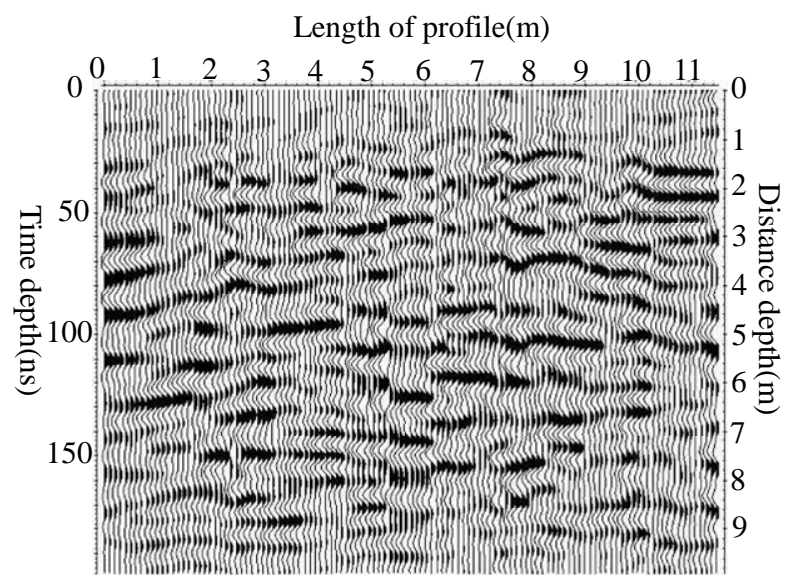

Fig.4 GPR image of typical joint fissure rock mass with water and mud

As daily observation in field, joints develop well in the central of the tunnel face, and mostly with distance of 0.4-0.2 meters. The surrounding rock is broken, weakly weathered. The fractures are expanded with fillings and rock surface is wet. From this the GPR detecting results are reliable.

\section{Detecting result of karst cave}

When the cave is filled with water, electromagnetic waves will be reflected around the cave, and the reflected waves are arc-shaped with stronger amplitude. When the cave is filled with rock pieces, similar with fracture rock mass, the amplitude of reflected waves get stronger and waveforms get disordered. When the cave is filled with clay, because of the absorption of electromagnetic waves, the reflected waves turn into weak amplitude and even disappeared. In Lirang tunnel, a karst cave was detected by GPR in the forecasting process and as shown in Figure 5.

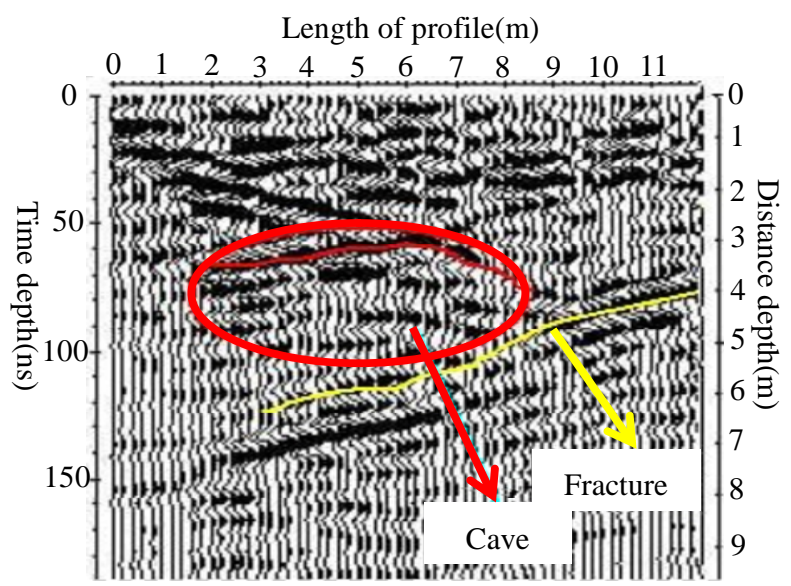

Fig.5 GPR image of typical karst cave

The practical experience of GPR method in Lirang tunnel above are typical and useful reference that the GPR method can detect adverse geological conditions of rock mass fracture zones, fracture and karst cave with positive effect.

\section{CONCLUSION}

Application of GPR in extra-long tunnel engineering of a variety of adverse geological conditions reveals its unique advantages,

(1) High frequency impulse electromagnetic waves are used with narrow wavelet electromagnetic pulse, and the wave frequency in the process of transmit and receive are high so that the GPR can do detecting continuously. The greatest advantage of GPR lies in its high resolution of detecting data.

(2) By use of high frequency transmitter, sampling and receiving time are short, the cycle test with different probing lines on the tunnel face is a save of human and material resources, detecting results can be gained in time that makes the tunnel construction on its rails.

(3) The results are intuitive that change rule of medium in front of the tunnel face can be reflected in the GPR images, especially in extra-long tunnel engineering of a variety of adverse geological 
conditions, as the construction carried forward and experience accumulated, the GPR method turns more mature in the process of geological forecasting, and the results get more reliable.

\section{REFERENCE}

[1] WANG Zhenyu, CHENG Weifeng, LIU Yue, ZHANG Yuhao. Synthetic advanced forecast technique based on geological logging for runnel face and ground penetrating radar [J]. Chinese Journal of Rock Mechanics and Engineering, 2010, 29(A02): 3549-3557.

[2] LING Tonghua, ZHANG Sheng, LI Shengran. Hilbert-Huang transform method for detection signal of tunnel geological prediction using ground penetrating radar [J]. Chinese Journal of Rock Mechanics and Engineering, 2012, 31(7): 1.

[3] GUO Liang, LI Juncai, ZHANG Zhicheng, CHENG Shoujin. Research on surrounding rock loose zone of tunnel under unsymmetrical loading with ground penetrating radar and its application [J]. Chinese Journal of Rock Mechanics and Engineering, 2011 (S1): 3009-3015.

[4] GAO Yang, ZHANG Qingsong, YUAN Xiaoshuai, XU Zhenhao, LIU Bin. Application of geological radar to geological forecast in karst tunnel [J]. JOURNAL OF SHANDONG UNIVERSITY(ENGINEERING SCIENCE), 2009, 39(4): 82r86.

[5] WU Jun, MAO Haihe, YING Song, XIA Caichu. Application of ground probing radar to shortterm geological forecast for tunnel construction [J]. Rock and Soil Mechanics, 2003 (S1): 154-157.

[6] Li Daxin. Ground penetrating radar method and application [M]. Beijing: Geology Publishing House, 1994. 